\title{
Weyl Fractional Integrals associated with the Transcendental Functions
}

\author{
Parul Gupta, Ashok Singh Shekhawat \\ Department of Mathematics, \\ Suresh Gyan Vihar University \\ Jaipur, Rajasthan, India
}

*Corresponding Author: Parul Gupta, Department of Mathematics, Suresh Gyan Vihar University

Jaipur, Rajasthan, India.Email: parul.gupta@live.in

\begin{abstract}
The present paper incorporates the systematic study of explicit form of generalized polynomial set which is defined by using Rodrigues type of formula during course of finding. Applications of Weyl fractional $q$-integral operator to various generalized basic hyper geometric functions including the basic analogue of Fox's H-function and some of its elementary properties have been investigated. We establish and derive the certain relations pertaining to the product of Fox's H-functions and general class of polynomials.
\end{abstract}

Keywords: General class of polynomial, Fox's H-function, Rodrigues formula, Fractional Integrals, Riemann Liouville operators.

\section{INTRODUCTION}

In this paper we introduce a unique Fractional Integral Operator of Weyl type and study that may be possible to express this integral operator as certain convolution with singular kernel of Riemann Liouville.

The General class of polynomial is defined by Srivastava [21] as-

$$
\mathrm{S}_{\mathrm{N}}^{\mathrm{M}}[\mathrm{x}]=\sum_{\mathrm{R}=0}^{[\mathrm{N} / \mathrm{M}]} \frac{(-\mathrm{N})_{\mathrm{MR}} \mathrm{V}_{\mathrm{N}, \mathrm{R}}}{\mathrm{R} !} \mathrm{x}^{\mathrm{R}}, \mathrm{N}=0,1,2, \ldots
$$

where $\mathrm{M}$ is an arbitrary positive integer and the coefficients $\mathrm{V}_{\mathrm{N}, \mathrm{R}}(\mathrm{N}, \mathrm{R} \geq 0)$ are arbitrary constants, real or complex. By suitably specializing the coefficients $\mathrm{V}_{\mathrm{N}, \mathrm{R}}$, the polynomials $\mathrm{S}_{\mathrm{N}}^{\mathrm{M}}[\mathrm{x}]$ can be reduced to the classical orthogonal polynomials such as Jacobi, Hermite, Legendre, Tchebycheff and Laguerre polynomials etc.

The generalized polynomial set is defined by following Rodrigues type of formula [15]:

$\mathrm{S}_{\mathrm{n}}^{\alpha, \beta, \tau}\left[\mathrm{x} ; \mathrm{c}, \mathrm{d}, \mathrm{q}, \mathrm{A}^{\prime}, \mathrm{B}^{\prime}, \mathrm{m}, \mathrm{g}, \ell\right]$

$=\left(\mathrm{A}^{\prime} \mathrm{x}+\mathrm{B}^{\prime}\right)^{-\alpha}\left(1-\tau \mathrm{x}^{\mathrm{c}}\right)^{-\beta / \tau} \mathrm{C}_{\mathrm{g}+\ell}^{\mathrm{m}+\mathrm{n}}\left[\left(\mathrm{A}^{\prime} \mathrm{x}+\mathrm{B}^{\prime}\right)^{\alpha+\mathrm{qn}}\left(1-\tau \mathrm{x}^{\mathrm{c}}\right)^{\beta / \tau+\mathrm{dn}}\right]$

with differential operator $\mathrm{C}_{\mathrm{g}, \ell}$ defined as

$$
\mathrm{C}_{\mathrm{g}, \ell}=\mathrm{x}^{\mathrm{g}}\left(\mathrm{g}+\mathrm{x} \frac{\mathrm{d}}{\mathrm{dx}}\right)
$$

The explicit form of this generalized polynomial set is as follows

$$
\begin{aligned}
& S_{n}^{\alpha, \beta, \tau}\left[x ; c, d, q, A^{\prime}, B^{\prime}, m, g, \ell\right] \\
& =\sum_{v^{\prime}, u^{\prime}, f^{\prime}, p} \theta\left(v^{\prime}, u^{\prime}, f^{\prime}, p\right) x^{R}\left(1-\tau x^{c}\right)^{d n-v^{\prime}}
\end{aligned}
$$


where

$$
\begin{aligned}
& \theta\left(\mathrm{v}^{\prime}, \mathrm{u}^{\prime}, \mathrm{f}^{\prime}, \mathrm{p}\right)=\mathrm{B}^{\prime(\mathrm{qn})} \mathrm{x}^{\ell(\mathrm{m}+\mathrm{n})} \frac{(-1)^{\mathrm{f}^{\prime}}\left(-\mathrm{f}^{\prime}\right)_{\mathrm{p}}(\alpha)_{\mathrm{f}}(-\alpha-\mathrm{qn})_{\mathrm{p}}\left(-\frac{\beta}{\tau}-\mathrm{dn}\right)_{\mathrm{v}^{\prime}}}{\mathrm{v}^{\prime} ! \mathrm{u}^{\prime} ! \mathrm{f}^{\prime} ! \mathrm{p} !\left(1-\alpha-\mathrm{f}^{\prime}\right)_{\mathrm{p}}} \\
& \cdot(-\tau)^{v^{\prime}}\left(\frac{p+g+r u^{\prime}}{\ell}\right)_{m+n}\left(\frac{A^{\prime}}{B^{\prime}}\right)^{f^{\prime}} \\
& \mathrm{R}=\ell(\mathrm{m}+\mathrm{n})+\mathrm{rv}^{\prime}+\mathrm{f}^{\prime}
\end{aligned}
$$

The series representation of Fox's H-function is given by

$$
\mathrm{H}_{\mathrm{P}_{1}, \mathrm{Q}_{1}}^{\mathrm{M}_{1}, \mathrm{~N}_{1}}\left[\mathrm{z}^{\prime} \mid \begin{array}{l}
\left(\mathrm{e}_{\mathrm{P}_{1}^{\prime}}^{\prime}, \mathrm{E}_{\mathrm{P}_{1}^{\prime}}^{\prime}\right) \\
\left({ }_{\mathrm{Q}_{1}}, \mathrm{~F}_{\mathrm{Q}_{1}}\right.
\end{array}\right]=\sum_{\mathrm{G}=0}^{\infty} \sum_{\mathrm{g}=1}^{\mathrm{M}_{1}} \frac{(-1)^{\mathrm{G}}}{\mathrm{G} ! \mathrm{F}_{\mathrm{g}^{\prime}}} \xi\left(\eta_{\mathrm{G}}^{\prime}\right) \mathrm{z}^{\prime \prime \mathrm{G}_{\mathrm{G}}^{\prime}}
$$

Also details can be read in (13).

The $\overline{\mathrm{H}}$-function defined by Inayat-Hussain [9] is

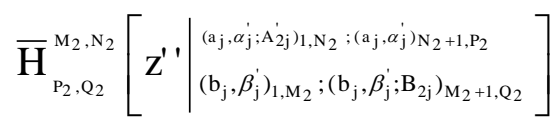

$$
\begin{aligned}
& =\frac{1}{2 \pi \mathrm{i}} \int_{-\mathrm{i} \omega}^{+\mathrm{i} \omega} \phi_{3}(\mathrm{~s}) \mathrm{z}^{\prime \prime \mathrm{s}} \mathrm{ds} .
\end{aligned}
$$

\section{Fractional Integrals:}

we will require Riemann - Liouville Integral defined as-

$$
\begin{aligned}
& \mathrm{D}^{-\mu}\{\mathrm{f}(\mathrm{x})\}={ }_{0} D_{X}^{-\mu}\{\mathrm{f}(\mathrm{x})\}= \\
& \frac{1}{\Gamma(\mu)} \int_{0}^{x}(x-w)^{\mu-1} f(w) d w, \quad \operatorname{Re}(\mu)>0 ; f \in J
\end{aligned}
$$

The classical Weyl Fractional integral operator of order $\mathrm{h}$ is defined as-

$$
W^{-h}\{f(x)\}={ }_{x} D_{\infty}^{-h}\{\mathrm{f}(\mathrm{x})\}=\frac{1}{\Gamma(\mathrm{h})} \int_{x}^{\infty}(\zeta-x)^{h-1} f(\zeta) d \zeta \operatorname{Re}(h)>0
$$

$x \geq 0, h>0$, and $f$ is a function belonging to $S(\mathrm{R})$, the Schwartzian space of functions.

\section{Main RESUlts}

In order to prove our Main Theorem, we need following results-

\section{Lemma 1}

(i) $\mathrm{P}_{2}, \mathrm{Q}_{2}, \mathrm{M}_{2}, \mathrm{~N}_{2}$ are integers such that $1 \leq \mathrm{M}_{2} \leq \mathrm{Q}_{2}, 0 \leq \mathrm{N}_{2} \leq \mathrm{P}_{2},\left(\alpha_{\mathrm{j}}\left(\mathrm{j}=1, \ldots, \mathrm{P}_{2}\right)\right.$, $\beta_{j}^{\prime}\left(j=1, \ldots, Q_{2}\right)$ are complex numbers.

(ii) $\operatorname{Re}\left(\mu^{\prime}\right)>\operatorname{Re}\left(h^{\prime}\right) ; \operatorname{Re}\left(h^{\prime}+\rho^{\prime} L+\sigma^{\prime} \eta_{G}^{\prime}+\lambda \frac{b_{j}}{\beta_{j}}\right)>0$, where $j=1, \ldots, M_{2}, M_{2}$ is a positive integer $\lambda \geq 0, \quad \mathrm{p}, \mathrm{v}^{\prime}=0,1, \ldots, \mathrm{n} ; \mathrm{z}^{\prime \prime} \geq 0, \sigma^{\prime} \geq 0$;

(iii) $\quad\left|\arg \mathrm{z}^{\prime}\right|<\frac{1}{2} \pi \Omega_{2}$, here

$$
\Omega_{2}=\sum_{j=1}^{M_{2}}\left|\beta_{j}^{\prime}\right|+\sum_{j=1}^{N_{2}}\left|A_{j} \alpha_{j}^{\prime}\right|-\sum_{j=M_{2}+1}^{Q_{2}}\left|B_{j} \beta_{j}^{\prime}\right|-\sum_{j=N_{2}+1}^{P_{2}}\left|\alpha_{j}^{\prime}\right|>0
$$


Then, we have

$$
\begin{aligned}
& \mathrm{W}^{\mathrm{h}^{\prime}-\mu^{\prime}}\left\{\mathrm{y}^{\prime-\mu^{\prime}} \mathrm{S}_{\mathrm{n}}^{\alpha, \beta, 0}\left[\mathrm{z}\left(\frac{\mathrm{x}^{\prime}}{\mathrm{y}^{\prime}}\right)^{\rho^{\prime}} ; \mathrm{c}, \mathrm{q}, \mathrm{A}^{\prime}, \mathrm{B}^{\prime}, \mathrm{m}, \mathrm{g}, \ell\right] \mathrm{S}_{\mathrm{N}}^{\mathrm{M}}\left[v\left(\frac{\mathrm{x}^{\prime}}{\mathrm{y}^{\prime}}\right)^{\mathrm{R}}\right] \mathrm{H}_{\mathrm{P}_{1}, \mathrm{Q}_{1}}^{\mathrm{M}_{1}, \mathrm{~N}_{1}}\left[\mathrm{z}^{\prime}\left(\frac{\mathrm{x}^{\prime}}{\mathrm{y}^{\prime}}\right)^{\sigma^{\prime}} \mid \begin{array}{l}
\left(\mathrm{e}_{\mathrm{P}_{1}^{\prime}}, \mathrm{E}_{\mathrm{P}_{1}}^{\prime}\right) \\
\left(\mathrm{f}_{\mathrm{Q}_{1}}, \mathrm{~F}_{\mathrm{Q}_{1}}\right)
\end{array}\right]\right. \\
& \left.\times \overline{\mathrm{H}}_{\mathrm{P}_{2}, \mathrm{Q}_{2}}^{\mathrm{M}_{2}, \mathrm{~N}_{2}}\left[\mathrm{z}^{\prime \prime}\left(\frac{\mathrm{x}^{\prime}}{\mathrm{y}^{\prime}}\right)^{\lambda} \mid \begin{array}{l}
\left(\mathrm{a}_{\mathrm{j}}, \alpha_{\mathrm{j}}^{\prime} ; \mathrm{A}_{2 \mathrm{j}}\right)_{1, \mathrm{~N}_{2}} ;\left(\mathrm{a}_{\mathrm{j}}, \alpha_{\mathrm{j}}^{\prime}\right)_{\mathrm{N}_{2}+1, \mathrm{P}_{2}} \\
\left(\mathrm{~b}_{\mathrm{j}}, \beta_{\mathrm{j}}^{\prime}\right)_{1, \mathrm{M}_{2}} ;\left(\mathrm{b}_{\mathrm{j}}, \beta_{\mathrm{j}}^{\prime} ; \mathrm{B}_{2 \mathrm{j}}\right)_{\mathrm{M}_{2}+1, \mathrm{Q}_{2}}
\end{array}\right]\right\} \\
& =\mathrm{y}^{\prime-\mathrm{h}^{\prime}} \sum \theta\left(\mathrm{f}^{\prime}, \mathrm{p}, \mathrm{u}^{\prime}, \mathrm{v}^{\prime}\right) \mathrm{z}^{\mathrm{L}} \sum_{\mathrm{G}=0}^{\infty} \sum_{\mathrm{g}^{\prime}=1}^{\mathrm{M}_{1}} \frac{(-1)^{\mathrm{G}}}{\mathrm{G} ! \mathrm{F}_{\mathrm{g}}} \xi\left(\eta_{\mathrm{G}}^{\prime}\right) \mathrm{z}^{\prime \eta_{\mathrm{G}}^{\prime}} \sum_{\mathrm{R}=0}^{[\mathrm{N} / \mathrm{M}]} \frac{(-\mathrm{N})_{\mathrm{M}_{\mathrm{R}}}}{\mathrm{R} !}\left(\frac{\mathrm{x}^{\prime}}{\mathrm{y}^{\prime}}\right)^{\rho^{\prime} \mathrm{L}+\sigma ; \eta_{\mathrm{G}}^{\prime}+\mathrm{R}} \\
& \times \overline{\mathrm{H}}_{\mathrm{P}_{2}, \mathrm{Q}_{2}}^{\mathrm{M}_{2}, \mathrm{~N}_{2}+1}\left[\mathrm{z}^{\prime}\left(\frac{\mathrm{x}^{\prime}}{\mathrm{y}^{\prime}}\right)^{\lambda} \mid \begin{array}{l}
\left(1-\mathrm{h}^{\prime}-\rho^{\prime} \mathrm{L}-\sigma^{\prime} \eta_{\mathrm{G}^{\prime}}^{\prime}-\mathrm{R}, \lambda ; 1\right),\left(\mathrm{a}_{\mathrm{j}}, \alpha_{\mathrm{j}}^{\prime} ; \mathrm{A}_{2 j}\right)_{1, \mathrm{~N}_{2}} ;\left(\mathrm{a}_{\mathrm{j}}, \alpha_{\mathrm{j}}^{\prime}\right)_{\mathrm{N}_{2}+1, \mathrm{P}_{2}} \\
\left(\mathrm{~b}_{\mathrm{j}}, \beta_{\mathrm{j}}^{\prime}\right)_{1, \mathrm{M}_{2}} ;\left(\mathrm{b}_{\mathrm{j}}, \beta_{\mathrm{j}}^{\prime} ; \mathrm{B}_{2 \mathrm{j}}\right)_{\mathrm{M}_{2}+1, \mathrm{Q}_{2}},\left(1-\mu^{\prime}-\rho^{\prime} \mathrm{L}-\sigma^{\prime} \eta_{\mathrm{G}^{\prime}}^{\prime}-\mathrm{R}, \lambda ; 1\right)
\end{array}\right]
\end{aligned}
$$

Proof:

In (1.2), taking $\mathrm{q}=\mathrm{g}=\mathrm{m}=0, \ell=-1$, and taking series representation of (1.2) and for the generalized polynomial set, general class of polynomial and Fox's H-function [13] respectively in the left hand side of (2.1), then expressing the Fox's $\overline{\mathrm{H}}$-function in Mellin-Barnes type contour integral and changing the order of summations and integrations justified under conditions stated, we find left hand side of (2.1)

$$
\begin{aligned}
& =\frac{1}{\Gamma\left(\mu^{\prime}-\mathrm{h}^{\prime}\right) 2 \pi \dot{\mathrm{d}}} \sum_{\mathrm{f}^{\prime}, \mathrm{p}, \mathrm{u}^{\prime}, \mathrm{v}^{\prime}} \phi_{1}\left(\mathrm{f}^{\prime}, \mathrm{p}, \mathrm{u}^{\prime}, \mathrm{v}^{\prime}\right) \sum_{\mathrm{G}=0}^{\infty} \sum_{\mathrm{g}=1}^{\mathrm{M}_{1}} \frac{(-1)^{\mathrm{G}}}{\mathrm{G} ! \mathrm{F}_{\mathrm{g}}} \xi\left(\eta_{\mathrm{G}}^{\prime}\right) \mathrm{z}^{\prime \eta_{\mathrm{G}}^{\prime}} \cdot \sum_{\mathrm{R}=0}^{[\mathrm{N} / \mathrm{M}]} \frac{(-\mathrm{N})_{\mathrm{M}_{\mathrm{R}}}}{\mathrm{R} !} \mathrm{z}^{\mathrm{L}} \cdot \\
& \quad \times \int_{-\mathrm{i} \omega}^{\mathrm{i} \omega} \phi_{3}(\mathrm{~s}) \mathrm{z}^{\prime \prime \mathrm{s}} \mathrm{x}^{\left(\rho^{\prime} \mathrm{L}+\sigma^{\prime} \eta_{\mathrm{G}}^{\prime}+\mathrm{R}+\lambda \mathrm{s}\right)}\left\{\int_{\mathrm{y}^{\prime}}^{\infty}\left(\psi-\mathrm{y}^{\prime}\right)^{\mu^{\prime}-\mathrm{h}^{\prime}-1} \psi^{\left.-\mu^{\prime}-\rho^{\prime} \mathrm{L}-\lambda \mathrm{s}-\mathrm{R}-\sigma^{\prime} \eta_{\mathrm{G}}^{\prime} \mathrm{d} \psi\right\} \mathrm{ds} .}\right.
\end{aligned}
$$

Now evaluating the inner $\psi$ integral in (2.2) with the help of known result (Beta Function) and the reinterpreting the resulting Mellin-Barnes contour integral in terms of $\overline{\mathrm{H}}$-function, we arrive at the desired result (2.1).

\section{Lemma 2.}

Under the conditions stated with Lemma 1, we have

$$
\begin{aligned}
& \int_{0}^{\infty} \mathrm{y}^{\prime^{-\mathrm{h}^{\prime}}} \sum_{\mathrm{f}^{\prime}, \mathrm{p}, \mathrm{u}^{\prime} \mathrm{v}^{\prime}} \theta\left(\mathrm{f}^{\prime}, \mathrm{p}, \mathrm{u}^{\prime}, \mathrm{v}^{\prime}\right) \mathrm{z}^{\mathrm{L}} \sum_{\mathrm{G}=0}^{\infty} \sum_{\mathrm{g}^{\prime}=1}^{\mathrm{M}_{1}} \frac{(-1)^{\mathrm{G}}}{\mathrm{G} ! \mathrm{F}_{\mathrm{g}^{\prime}}} \xi\left(\eta_{\mathrm{G}}^{\prime}\right) \mathrm{z}^{\mathrm{z}^{\prime} \eta_{\mathrm{G}}^{\prime}} \sum_{\mathrm{R}=0}^{[\mathrm{N} / \mathrm{M}]} \frac{(-\mathrm{N})_{\mathrm{M}_{\mathrm{R}}}}{\mathrm{R} !}\left(\frac{\mathrm{x}^{\prime}}{\mathrm{y}^{\prime}}\right)^{\rho^{\prime} \mathrm{L}+\sigma^{\prime} \eta_{\mathrm{G}}^{\prime}+\mathrm{R}} \\
& \times \overline{\mathrm{H}}_{\mathrm{P}_{2}+1, \mathrm{Q}_{2}+1}^{\mathrm{M}_{2}, \mathrm{~N}_{2}+1}\left[\mathrm{z}^{\prime \prime}\left(\frac{\mathrm{x}^{\prime}}{\mathrm{y}^{\prime}}\right)^{\lambda} \begin{array}{l}
\left(1-\mathrm{h}^{\prime}-\rho^{\prime} \mathrm{L}-\sigma^{\prime} \eta_{\mathrm{G}}^{\prime}-\mathrm{R}, \lambda ; 1\right),\left(\mathrm{a}_{\mathrm{j}}, \alpha_{\mathrm{j}}^{\prime} ; \mathrm{A}_{2 \mathrm{2}}\right)_{1, \mathrm{~N}_{2}} ;\left(\mathrm{a}_{\mathrm{j}}, \alpha_{\mathrm{j}}^{\prime}\right)_{\mathrm{N}_{2}+1, \mathrm{P}_{2}} \\
\left(\mathrm{~b}_{\mathrm{j}}, \beta_{\mathrm{j}}^{\prime}\right)_{1, \mathrm{M}_{2}} ;\left(\mathrm{b}_{\mathrm{j}}, \beta_{\mathrm{j}}^{\prime} ; \mathrm{B}_{\mathrm{j}}\right)_{\mathrm{M}_{2}+1, \mathrm{Q}_{2}},\left(1-\mu^{\prime}-\rho^{\prime} \mathrm{L}-\sigma^{\prime} \eta_{\mathrm{G}}-\mathrm{R}, \lambda ; 1\right)
\end{array}\right] \mathrm{f}\left(\mathrm{y}^{\prime}\right) \mathrm{dy^{ \prime }} \\
& =\int_{0}^{\infty} \zeta^{-\mu^{\prime}} \mathrm{S}_{\mathrm{n}}^{\alpha, \beta, 0}\left[\mathrm{z}\left(\frac{\mathrm{x}^{\prime}}{\zeta}\right)^{\rho^{\prime}} ; \mathrm{c}, \mathrm{q}, \mathrm{A}^{\prime}, \mathrm{B}^{\prime}, \mathrm{g}, \ell\right] \mathrm{S}_{\mathrm{N}}^{\mathrm{M}}\left[v\left(\frac{\mathrm{x}^{\prime}}{\zeta}\right)^{\mathrm{R}}\right] \mathrm{H}_{\mathrm{P}_{1}, \mathrm{Q}_{1}}^{\mathrm{M}_{1}, \mathrm{~N}_{1}}\left[\mathrm{z}^{\prime}\left(\frac{\mathrm{x}^{\prime}}{\zeta}\right)^{\sigma^{\prime}} \mid \begin{array}{l}
\left(\mathrm{e}_{\mathrm{P}_{1}}^{\prime}, \mathrm{E}_{\mathrm{P}_{\mathrm{P}}}^{\prime}\right) \\
\left(\mathrm{f}_{\mathrm{Q}_{1}}, \mathrm{~F}_{\mathrm{Q}_{1}}\right)
\end{array}\right] \\
& \times \overline{\mathrm{H}}_{\mathrm{P}_{2}, \mathrm{Q}_{2}}^{\mathrm{M}_{2}, \mathrm{~N}_{2}}\left[\mathrm{z}^{\prime \prime}\left(\frac{\mathrm{x}^{\prime}}{\zeta}\right)^{\lambda \mid} \mid \begin{array}{l}
\left(\mathrm{a}_{\mathrm{j}}, \alpha_{\mathrm{j}}^{\prime} ; \mathrm{A}_{\mathrm{j}}\right)_{1, \mathrm{~N}_{2}} ;\left(\mathrm{a}_{\mathrm{j}}, \alpha_{\mathrm{j}}^{\prime}\right)_{\mathrm{N}_{2}+1, \mathrm{P}_{2}} \\
\left(\mathrm{~b}_{\mathrm{j}}, \beta_{\mathrm{j}}^{\prime}\right)_{1, \mathrm{M}_{2}} ;\left(\mathrm{b}_{\mathrm{j}}, \beta_{\mathrm{j}}^{\prime} ; \mathrm{B}_{2 \mathrm{j}}\right)_{\mathrm{M}_{2}+1, \mathrm{Q}}
\end{array}\right] \mathrm{D}^{\left(\mathrm{h}^{\prime}-\mu^{\prime}\right)} \mathrm{f}(\zeta) \mathrm{d} \zeta
\end{aligned}
$$


provided $\mathrm{f} \in \zeta$ and $\mathrm{x}^{\prime}>0$.

\section{Proof:}

Let $\Delta$ denotes the left hand side, then using Lemma - 1 and applying

$$
\omega^{-\mathrm{h}^{\prime}}\{\phi(\mathrm{x})\}=\frac{1}{\Gamma\left(\mathrm{h}^{\prime}\right)} \int_{\mathrm{x}^{\prime}}^{\omega}\left(\zeta-\mathrm{x}^{\prime}\right)^{\mathrm{h}^{\prime}-1} \mathrm{f}(\zeta) \mathrm{d} \zeta
$$

we get

$$
\begin{aligned}
& \Delta=\int_{0}^{\infty}\left\{\frac{1}{\Gamma\left(\mu^{\prime}-\mathrm{h}^{\prime}\right)} \int_{\mathrm{y}^{\prime}}^{\infty}\left(\zeta-\mathrm{y}^{\prime}\right)^{\mu^{\prime}-\mathrm{h}^{\prime}-1} \zeta^{-\mu^{\prime}}\right. \\
& \times \mathrm{S}_{\mathrm{n}}^{\alpha, \beta, 0}\left[\mathrm{z}\left(\frac{\mathrm{x}^{\prime}}{\zeta}\right)^{\rho^{\prime}} ; \mathrm{c}, \mathrm{q}, \mathrm{A}^{\prime}, \mathrm{B}^{\prime}, \mathrm{g}, \ell\right] \mathrm{S}_{\mathrm{N}}^{\mathrm{M}}\left[\mathrm{n}\left(\frac{\mathrm{x}^{\prime}}{\zeta}\right)^{\mathrm{R}}\right] \mathrm{H}_{\mathrm{P}_{1}, \mathrm{Q}_{1}}^{\mathrm{M}_{1}, \mathrm{~N}_{1}}\left[\mathrm{z}^{\prime}\left(\frac{\mathrm{x}^{\prime}}{\zeta}\right)^{\sigma^{\prime}} \mid \begin{array}{l}
\left(\mathrm{e}_{\mathrm{P}_{1}}, \mathrm{E}_{\mathrm{P}_{1}}\right) \\
\left(\mathrm{f}_{\mathrm{Q}_{1}}, \mathrm{~F}_{\mathrm{Q}_{1}}\right)
\end{array}\right] \\
& \left.\times \overline{\mathrm{H}}_{\mathrm{P}_{2}, \mathrm{Q}_{2}}^{\mathrm{M}_{2}, \mathrm{~N}_{2}}\left[\mathrm{z}^{\prime \prime}\left(\frac{\mathrm{x}^{\prime}}{\zeta}\right)^{\lambda} \mid \begin{array}{l}
\left(\mathrm{a}_{\mathrm{j}}, \alpha_{\mathrm{j}}^{\prime} ; \mathrm{A}_{\mathrm{j}}\right)_{1, \mathrm{~N}_{2}} ;\left(\mathrm{a}_{\mathrm{j}}, \alpha_{\mathrm{j}}^{\prime}\right)_{\mathrm{N}_{2}+1, \mathrm{P}_{2}} \\
\left(\mathrm{~b}_{\mathrm{j}}, \beta_{\mathrm{j}}^{\prime}\right)_{1, \mathrm{M}_{2}} ;\left(\mathrm{b}_{\mathrm{j}}, \beta_{\mathrm{j}}^{\prime} ; \mathrm{B}_{2 \mathrm{j}}\right)_{\mathrm{M}_{2}+1, \mathrm{Q}_{2}}
\end{array}\right] \mathrm{d} \zeta\right\} \mathrm{f}\left(\mathrm{y}^{\prime}\right) \mathrm{dy^{ \prime }}
\end{aligned}
$$

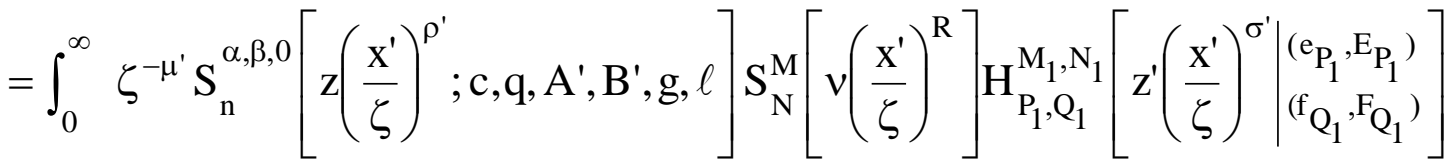

$$
\begin{aligned}
& \times \overline{\mathrm{H}}_{\mathrm{P}_{2}, \mathrm{Q}_{2}}^{\mathrm{M}_{2}, \mathrm{~N}_{2}}\left[\mathrm{z}^{\prime \prime}\left(\frac{\mathrm{x}^{\prime}}{\zeta}\right)^{\lambda \mid} \mid \begin{array}{c}
\left(\mathrm{a}_{\mathrm{j}}, \alpha_{\mathrm{j}}^{\prime} ; \mathrm{A}_{\mathrm{j}}\right)_{1, \mathrm{~N}_{2}} ;\left(\mathrm{a}_{\mathrm{j}}, \alpha_{\mathrm{j}}^{\prime}\right)_{\mathrm{N}_{2}+1, \mathrm{P}_{2}} \\
\left(\mathrm{~b}_{\mathrm{j}}, \beta_{\mathrm{j}}^{\prime}\right)_{1, \mathrm{M}_{2}} ;\left(\mathrm{b}_{\mathrm{j}}, \beta_{\mathrm{j}}^{\prime} ; \mathrm{B}_{\mathrm{j}}\right)_{\mathrm{M}_{2}+1, \mathrm{Q}_{2}}
\end{array}\right]\left\{\int_{\mathrm{y}^{\prime}}^{\omega} \frac{\left(\zeta-\mathrm{y}^{\prime}\right)^{\mu^{\prime}-\mathrm{h}^{\prime}-1}}{\Gamma\left(\mu^{\prime}-\mathrm{h}^{\prime}\right)} \mathrm{f}\left(\mathrm{y}^{\prime}\right) \mathrm{dy^{ \prime }}\right\} \mathrm{d} \zeta
\end{aligned}
$$

it has been assumed that change of order of integration is permissible as in Lemma 1.

\section{MAIN THEOREM}

$$
\text { If } \mathrm{f} \in \mathrm{J}, \mathrm{D}^{\mu-\mathrm{h}^{\prime}}\{\mathrm{f}\} \text { exist, } \lambda>0, \mathrm{x}>0,|\arg \mathrm{w}|<\frac{\pi}{2} \Omega_{2}, \Omega_{2}>0, \operatorname{Re}(\mu)>\operatorname{Re}\left(\mathrm{h}^{\prime}\right)>0 \text {, }
$$

then the solution of integral equation-

$$
\int_{0}^{\omega} y^{\prime-\mu^{\prime}} u\left(\frac{x^{\prime}}{y^{\prime}}\right) f\left(y^{\prime}\right) d y^{\prime}=g\left(x^{\prime}\right), x^{\prime}>0
$$

is given by

$$
\mathrm{f}\left(\mathrm{x}^{\prime}\right)=\frac{\lambda}{2 \pi \mathrm{i}} \mathrm{x}^{\mu^{\prime}-1} \lim _{\gamma \rightarrow \infty} \int_{\mathrm{C}-\mathrm{i} \gamma}^{\mathrm{c}+\mathrm{i} \gamma} \frac{\mathrm{x}^{\prime-\mathrm{s}} \phi(\mathrm{s})}{\theta\left(\mathrm{s}, \mathrm{z}, \mathrm{z}^{\prime}, v \cdot \mathrm{z}^{\prime}\right)} \mathrm{ds}
$$

here

$$
\phi(s)=\int_{0}^{\omega} x^{\prime s-1} g(x) d x
$$

and

$$
\theta\left(s, z, z^{\prime}, z^{\prime \prime}, v\right)=\sum_{f, p, u^{\prime}, v^{\prime}} \phi_{1}\left(f^{\prime}, p, u^{\prime}, v^{\prime}\right) z^{L} \sum_{R=0}^{[N / M]} \frac{(-N)}{R !} \sum_{G=0}^{\omega} \sum_{g=1}^{M_{1}} \frac{(-1)^{G}}{G ! F} \xi\left(\eta_{G}^{\prime}\right) z^{\prime} \eta_{G}^{\prime}
$$




$$
\times \phi_{3}\left(\frac{-\rho^{\prime} \mathrm{L}-\mathrm{s}-\sigma^{\prime} \eta_{\mathrm{G}}^{\prime}-\mathrm{tR}}{\lambda}\right) \mathrm{z}^{\prime \prime}\left(\frac{\mathrm{s}+\rho^{\prime} \mathrm{L}+\sigma^{\prime} \eta_{\mathrm{G}}^{\prime}+\mathrm{R}}{\lambda}\right),
$$

provided that

$$
-\min _{1 \leq \mathrm{j} \leq \mathrm{M}_{2}} \operatorname{Re}\left(\frac{\mathrm{b}_{\mathrm{j}}}{\beta_{2 \mathrm{j}}}\right)<\operatorname{Re}\left(\frac{\mathrm{s}+\rho^{\prime} \mathrm{L}+\sigma^{\prime} \eta_{\mathrm{G}}^{\prime}+\mathrm{R}}{\lambda}\right)<\min _{1 \leq \mathrm{j} \leq \mathrm{N}_{2}} \operatorname{Re}\left\{\frac{\left(1-\mathrm{a}_{\mathrm{j}}\right)}{\mathrm{A}_{2 \mathrm{j}}}\right\}
$$

where $\mathrm{L}=\ell \mathrm{n}+\mathrm{p}+\mathrm{c} \mathrm{v}^{\prime}: \mathrm{p}, \mathrm{v}^{\prime}=0,1, \ldots, \mathrm{n}$.

\section{Proof:}

Replacing $D^{\mu^{\prime}-h^{\prime}}(f)$ in left hand side of (2.3) of Lemma - 2, we have

$$
\begin{aligned}
& g\left(x^{\prime}\right)=\int_{0}^{\omega} y^{\prime-h^{\prime}} \sum_{f, p, u^{\prime}, v^{\prime}} \theta\left(f^{\prime}, p, u^{\prime}, v^{\prime}\right) z^{L} \sum_{R=0}^{[N / M]} \frac{(-N)_{R}}{R !} \sum_{G=0}^{\omega} \sum_{g=1}^{M_{1}} \frac{(-1)^{G}}{G ! F_{g}} \xi\left(\eta_{G}^{\prime}\right) z^{\prime \eta_{G}^{\prime}} \\
& \times\left(\frac{x^{\prime}}{y^{\prime}}\right)^{\rho^{\prime} L+\sigma^{\prime} \eta_{G^{\prime}}^{\prime}+R} \bar{H}_{P_{2}, Q_{2}}^{M_{2}, N_{2}}\left[\left.z^{\prime \prime}\left(\frac{x^{\prime}}{y^{\prime}}\right)^{\lambda \mid}\right|_{\left(b_{j}, \beta_{j}^{\prime}\right)_{1, M_{2}},\left(b_{j}, \beta_{j}^{\prime} ; B_{2 j}\right)_{M_{2}+1, Q_{2}},\left(1-\mu^{\prime}-\sigma^{\prime} \eta_{G}^{\prime}-\rho^{\prime} L-R-R, \lambda ; 1\right)} ^{\left.(1)-\sigma^{\prime} \eta_{G}^{\prime}, \lambda ; 1\right),\left(a_{j}, \alpha_{j}^{\prime} ; A_{j}\right)_{1, N_{2}},\left(a_{j}, \alpha_{j}^{\prime}\right)_{N_{2}+1, P_{2}}}\right] \\
& \times \mathrm{D}^{\mu^{\prime}-\mathrm{h}^{\prime}}\left\{\mathrm{f}\left(\mathrm{y}^{\prime}\right)\right\} d \mathrm{y}^{\prime} .
\end{aligned}
$$

Now taking Mellin transform of both sides by multiplying both sides of (2.10) by $\mathrm{x}^{\mathrm{s}-1}$ and integrating with respect to $\mathrm{x}^{\prime}$ from 0 to $\mathrm{w}_{1}$, we have

$$
\begin{aligned}
& \phi\left(x^{\prime}\right)=\int_{0}^{\omega} y^{\prime^{\prime} h^{\prime}} \sum_{\left(f, p, u^{\prime}, v^{\prime}\right)} \theta\left(f^{\prime}, p, u^{\prime}, v^{\prime}\right) z^{L} \sum_{R=0}^{[N / M]} \frac{(-N)_{R}}{R !} \sum_{G=0}^{\omega} \sum_{g=1}^{M_{1}} \frac{(-1)^{G}}{G ! F_{g}} \xi\left(\eta_{G}^{\prime}\right) z^{\prime^{\prime} \eta_{G}^{\prime}}
\end{aligned}
$$

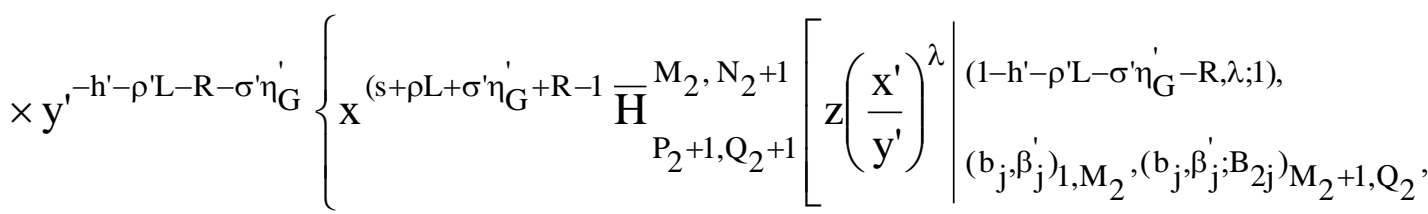

$$
\begin{aligned}
& \left.\left.\underset{\left(1-\mu-\rho^{\prime} L-\sigma^{\prime} \eta_{G}^{\prime}-R, \lambda ; 1\right.}{\left(a_{j}, \alpha_{j}^{\prime} ; A_{j}\right)_{1, N_{2}},\left(a_{j}, \alpha_{j}\right)_{N_{2}+1, P_{2}}}\right] d x^{\prime}\right\} D^{\left(\mu^{\prime}-h^{\prime}\right)}\left\{f\left(y^{\prime}\right)\right\} d y^{\prime}
\end{aligned}
$$

where it has been assumed that the change of order of integration is permissible under the stated conditions.

On evaluating the inner integral, (2.11) reduces to

$$
\frac{\Gamma\left(\mu^{\prime}-\mathrm{s}\right) \phi(\mathrm{s})}{\Gamma\left(\mathrm{h}^{\prime}-\mathrm{s}\right) \theta\left(\mathrm{s}, \mathrm{z}, \mathrm{z}^{\prime}, \mathrm{z}^{\prime \prime}, v\right)}=\mathrm{M}_{\mathrm{t}}\left[\mathrm{y}^{\prime{ }^{\prime}-\mathrm{h}^{\prime}} \mathrm{D}^{\mu^{\prime}-\mathrm{h}^{\prime}}\left\{\mathrm{f}\left(\mathrm{y}^{\prime}\right)\right\}: \mathrm{s}\right]
$$

which on applying inversion theorem gives

$$
\mathrm{D}^{\mu^{\prime}-\mathrm{h}^{\prime}}\left\{\mathrm{f}\left(\mathrm{y}^{\prime}\right)\right\}=\frac{\lambda}{2 \pi \mathrm{i}} \lim _{\gamma \rightarrow \infty} \int_{\mathrm{c}-\mathrm{i} \gamma}^{\mathrm{c}+\mathrm{i} \gamma} \mathrm{y}^{\mathrm{h}^{\prime}-\mathrm{s}^{\prime}-1} \frac{\Gamma\left(\mu{ }^{\prime}-\mathrm{s}\right) \phi(\mathrm{s}) \mathrm{ds}}{\Gamma\left[\left(\mathrm{h}^{\prime}-\mathrm{s}\right) \cdot \theta\left(\mathrm{s}, \mathrm{z}, \mathrm{z}^{\prime}, \mathrm{z}^{\prime}, v\right)\right]}
$$


Now operating upon both the sides of $(2.13)$ by $\mathrm{D}^{\mathrm{h}^{\prime}-\mu^{\prime}}$ and change the order of integration permissible under the conditions stated, we obtain

$$
\begin{aligned}
\mathrm{f}\left(\mathrm{y}^{\prime}\right)= & \frac{\lambda}{\Gamma\left(\mu^{\prime}-\mathrm{h}^{\prime}\right)} \lim _{\gamma \rightarrow \infty} \int_{\mathrm{c}-\mathrm{i} \gamma}^{\mathrm{c}+\mathrm{i} \gamma} \frac{\Gamma\left(\mu^{\prime}-\mathrm{s}\right)}{\Gamma\left(\mathrm{h}^{\prime}-\mathrm{s}\right) \theta\left(\mathrm{s}, \mathrm{z}, \mathrm{z}^{\prime}, \mathrm{z}^{\prime \prime} v\right)} \\
& \times\left\{\int_{0}^{\mathrm{y}^{\prime}} \xi^{\mathrm{h}^{\prime}-\mathrm{s}-1}\left(\mathrm{y}^{\prime}-\zeta\right)^{\mu^{\prime}-\mathrm{h}^{\prime}-1} \mathrm{~d} \zeta\right\} \phi(\mathrm{s}) \mathrm{ds}
\end{aligned}
$$

By evaluating inner integral of (2.14) by the definition of Beta function, we get the required result (2.6).

\section{Interesting Special CaSes}

(1) If we set $A_{2}=1, B_{2}=q=0, \ell=-1$ in (2.1), (2.3),(2.6), we obtain the following results

(i)

$$
\begin{aligned}
& \mathrm{z}^{\prime \prime \mathrm{h}^{\prime}-\mu^{\prime}}\left\{\mathrm{y}^{-\mu^{\prime}} \mathrm{H}_{\mathrm{n}}^{\left(\mathrm{r}^{\prime}\right)}\left[\mathrm{z}\left(\frac{\mathrm{x}^{\prime}}{\mathrm{y}^{\prime}}\right)^{\rho^{\prime}}, \alpha, \beta\right] \mathrm{S}_{\mathrm{N}}^{\mathrm{M}}\left[v\left(\frac{\mathrm{x}^{\prime}}{\mathrm{y}^{\prime}}\right)^{\mathrm{R}}\right] \mathrm{H}_{\mathrm{P}_{1}, \mathrm{Q}_{1}}^{\mathrm{M}_{1}, \mathrm{~N}_{1}}\left[\mathrm{z}^{\prime}\left(\frac{\mathrm{x}^{\prime}}{\mathrm{y}^{\prime}}\right)^{\sigma^{\prime}} \mid \begin{array}{l}
\left(\mathrm{e}_{\mathrm{P}_{1}}, \mathrm{E}_{\mathrm{P}_{1}}\right) \\
\left(\mathrm{f}_{\mathrm{Q}_{1}}, \mathrm{~F}_{\mathrm{Q}_{1}}\right.
\end{array}\right]\right. \\
& \left.\times \overline{\mathrm{H}}_{\mathrm{P}_{2}, \mathrm{Q}_{2}}^{\mathrm{M}_{2}, \mathrm{~N}_{2}}\left[\mathrm{z}^{\prime \prime}\left(\frac{\mathrm{x}^{\prime}}{\mathrm{y}^{\prime}}\right)^{\lambda \mid} \mid \begin{array}{c}
\left(\mathrm{a}_{\mathrm{j}}, \alpha_{\mathrm{j}}^{\prime} ; \mathrm{A}_{\mathrm{j}}\right)_{1, \mathrm{~N}_{2}} ;\left(\mathrm{a}_{\mathrm{j}}, \alpha_{\mathrm{j}}^{\prime}\right)_{\mathrm{N}_{2}+1, \mathrm{P}_{2}} \\
\left(\mathrm{~b}_{\mathrm{j}}, \beta_{\mathrm{j}}^{\prime}\right)_{1, \mathrm{M}_{2}} ;\left(\mathrm{b}_{\mathrm{j}}, \beta_{\mathrm{j}}^{\prime} ; \mathrm{B}_{\mathrm{j}}\right)_{\mathrm{M}_{2}+1, \mathrm{Q}_{2}}
\end{array}\right]\right\} \\
& =\mathrm{y}^{\prime}-\mathrm{h}^{\prime} \sum_{\mathrm{v}^{\prime}=0}^{\mathrm{n}} \sum_{\mathrm{u}^{\prime}=0}^{\mathrm{v}^{\prime}} \frac{(-\mathrm{v} \cdot)_{\mathrm{u}^{\prime}}\left(-\alpha-\mathrm{ru}^{\prime}\right)}{\mathrm{u}^{\prime} ! \mathrm{v}^{\prime} !} \beta^{\mathrm{v}^{\prime}} \mathrm{z}^{\mathrm{rv}-\mathrm{n}} \sum_{\mathrm{R}=0}^{[\mathrm{N} / \mathrm{M}]} \frac{(-\mathrm{N})_{\mathrm{R}}}{\mathrm{R} !} \\
& \sum_{G=0}^{\omega} \sum_{g^{\prime}=1}^{M_{1}} \frac{(-1)^{G}}{G ! F_{g^{\prime}}} \xi\left(\eta_{G}^{\prime}\right) z^{\prime \eta_{G}^{\prime}}\left(\frac{x^{\prime}}{y^{\prime}}\right)^{\sigma^{\prime} \eta_{G^{\prime}}+\rho^{\prime}\left(r v^{\prime}-n\right)} \\
& \times \overline{\mathrm{H}}_{\mathrm{P}_{2}+1, \mathrm{Q}_{2}+1}^{\mathrm{M}_{2}, \mathrm{~N}_{2}+1}\left[\mathrm{z}^{\prime \prime}\left(\frac{\mathrm{x}^{\prime}}{\mathrm{y}^{\prime}}\right)^{\lambda} \mid \begin{array}{l}
\left(1-\mathrm{h}^{\prime}-\sigma^{\prime} \eta_{\mathrm{G}^{\prime}}^{\prime}-\rho^{\prime} r v^{\prime}-\mathrm{R}-\rho^{\prime} \mathrm{n}, \lambda ; 1\right),\left(\mathrm{a}_{\mathrm{j}}, \alpha_{\mathrm{j}}^{\prime} ; \mathrm{A}_{2 j}\right)_{1, \mathrm{~N}_{2}} ;\left(\mathrm{a}_{\mathrm{j}}, \alpha_{\mathrm{j}}^{\prime}\right)_{\mathrm{N}_{2}+1, \mathrm{P}_{2}} \\
\left(\mathrm{~b}_{\mathrm{j}}, \beta_{\mathrm{j}}^{\prime}\right)_{1, \mathrm{M}_{2}} ;\left(\mathrm{b}_{\mathrm{j}}, \beta_{\mathrm{j}}^{\prime} ; \mathrm{B}_{\mathrm{j}}\right)_{\mathrm{M}_{2}+1, \mathrm{Q}_{2}},\left(1-\mu^{\prime}-\sigma^{\prime} \eta_{\mathrm{G}^{\prime}}^{\prime}-\rho^{\prime} r v^{\prime}-\mathrm{R}+\rho^{\prime} \mathrm{n}, \lambda^{\prime} ; 1\right)
\end{array}\right] \\
& \int_{0}^{\mathrm{w}} \rho^{-\mu^{\prime}}\left[\mathrm{z}\left(\frac{\mathrm{x}^{\prime}}{\zeta}\right)^{\rho^{\prime}}+\alpha, \beta\right] \mathrm{H}_{\mathrm{P}_{1}, \mathrm{Q}_{1}}^{\mathrm{M}_{1}, \mathrm{~N}_{1}}\left[\mathrm{z}^{\prime} \mathrm{z}\left(\frac{\mathrm{x}^{\prime}}{\zeta}\right)^{\sigma^{\prime}} \mid \begin{array}{l}
\left(\mathrm{e}_{\mathrm{P}_{1}}, \mathrm{E}_{\mathrm{P}_{1}}\right) \\
\left(\mathrm{f}_{\mathrm{Q}_{1}}, \mathrm{~F}_{\mathrm{Q}_{1}}\right.
\end{array}\right] \mathrm{S}_{\mathrm{N}}^{\mathrm{M}}\left[v \mathrm{z}\left(\frac{\mathrm{x}^{\prime}}{\zeta}\right)^{\mathrm{R}}\right] \\
& \times \overline{\mathrm{H}}_{\mathrm{P}_{2}, \mathrm{Q}_{2}}^{\mathrm{M}_{2}, \mathrm{~N}_{2}}\left[\mathrm{z}^{\prime \prime}\left(\frac{\mathrm{x}}{\zeta}\right)^{\lambda \mid} \mid \begin{array}{l}
\left(\mathrm{a}_{\mathrm{j}}, \alpha_{\mathrm{j}}^{\prime} ; \mathrm{A}_{\mathrm{j}}\right)_{1, \mathrm{~N}_{2}} ;\left(\mathrm{a}_{\mathrm{j}}, \alpha_{\mathrm{j}}^{\prime}\right)_{\mathrm{N}_{2}+1, \mathrm{P}_{2}} \\
\left(\mathrm{~b}_{\mathrm{j}}, \beta_{\mathrm{j}}^{\prime}\right)_{1, \mathrm{M}_{2}} ;\left(\mathrm{b}_{\mathrm{j}}, \beta_{\mathrm{j}}^{\prime} ; \mathrm{B}_{\mathrm{j}}\right)_{\mathrm{M}_{2}+1, \mathrm{Q}_{2}}
\end{array}\right] \mathrm{D}^{\mathrm{h}^{\prime}-\mu^{\prime}}\{\mathrm{f}(\zeta)\} \mathrm{d} \zeta \\
& =\int_{0}^{\omega} \mathrm{y}^{\prime-\mathrm{h}^{\prime}} \sum_{\mathrm{v}^{\prime}=0}^{\mathrm{n}} \sum_{\mathrm{u}^{\prime}=0}^{\mathrm{v}^{\prime}} \frac{(-\mathrm{v} .)_{\mathrm{u}^{\prime}}\left(-\alpha-\mathrm{ru}^{\prime}\right)}{\mathrm{u}^{\prime} ! \mathrm{v}^{\prime} !} \beta^{\mathrm{v}^{\prime}} \mathrm{z}^{\mathrm{rv}-\mathrm{n}} \sum_{\mathrm{R}=0}^{[\mathrm{N} / \mathrm{M}]} \frac{(-\mathrm{N})_{\mathrm{R}}}{\mathrm{R} !} \mathrm{A}_{1, \mathrm{~N}, \mathrm{R}} \\
& \sum_{G=0}^{\omega} \sum_{g^{\prime}=1}^{M_{1}} \frac{(-1)^{G}}{G ! F_{g^{\prime}}} \xi\left(\eta_{G}^{\prime}\right) z^{\eta_{G}^{\prime}}\left(\frac{x^{\prime}}{y^{\prime}}\right)^{\sigma^{\prime} \eta_{G^{\prime}}+\rho^{\prime}\left(r v^{\prime}-n\right)}
\end{aligned}
$$




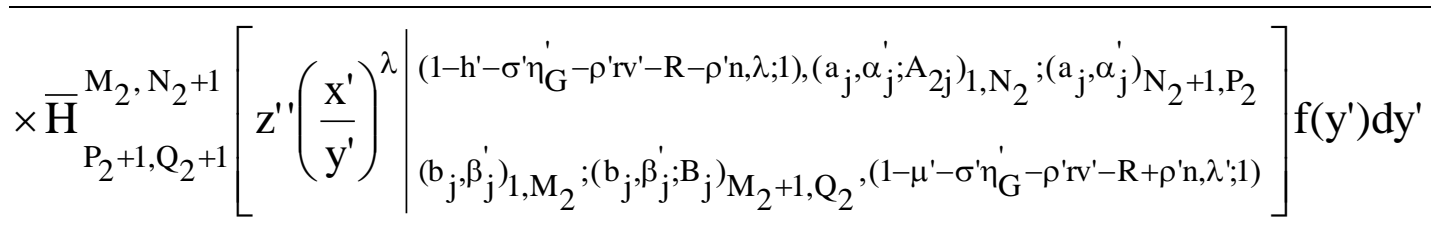

(iii)

$$
\begin{aligned}
& \int_{0}^{\mathrm{w}} \mathrm{y}^{\prime-\mu^{\prime}} \mathrm{H}_{\mathrm{n}}^{\left(\mathrm{r}^{\prime}\right)}\left[\mathrm{z}\left(\frac{\mathrm{x}^{\prime}}{\zeta}\right)^{\rho^{\prime}}+\alpha, \beta\right] \mathrm{S}_{\mathrm{N}}^{\mathrm{M}}\left[v\left(\frac{\mathrm{x}^{\prime}}{\zeta}\right)^{\mathrm{R}}\right] \mathrm{H}_{\mathrm{P}_{1}, \mathrm{Q}_{1}}^{\mathrm{M}_{1}, \mathrm{~N}_{1}}\left[\mathrm{z}^{\prime}\left(\frac{\mathrm{x}^{\prime}}{\zeta}\right)^{\sigma^{\prime}} \mid \begin{array}{l}
\left(\mathrm{e}_{\mathrm{P}_{1}}, \mathrm{E}_{\mathrm{P}_{1}}\right) \\
\left(\mathrm{f}_{\mathrm{Q}_{1}}, \mathrm{~F}_{\mathrm{Q}_{1}}\right.
\end{array}\right] \\
& \times \overline{\mathrm{H}}_{\mathrm{P}_{2}, \mathrm{Q}_{2}}^{\mathrm{M}_{2}, \mathrm{~N}_{2}}\left[\mathrm{z}^{\prime \prime}\left(\frac{\mathrm{x}^{\prime}}{\zeta}\right)^{\lambda} \mid \begin{array}{l}
\left(\mathrm{a}_{\mathrm{j}}, \alpha_{\mathrm{j}}^{\prime} ; \mathrm{A}_{\mathrm{j}}\right)_{1, \mathrm{~N}_{2}} ;\left(\mathrm{a}_{\mathrm{j}}, \alpha_{\mathrm{j}}^{\prime}\right)_{\mathrm{N}_{2}+1, \mathrm{P}_{2}} \\
\left(\mathrm{~b}_{\mathrm{j}}, \beta_{\mathrm{j}}^{\prime}\right)_{1, \mathrm{M}_{2}} ;\left(\mathrm{b}_{\mathrm{j}}, \beta_{\mathrm{j}}^{\prime} ; \mathrm{B}_{\mathrm{j}}\right)_{\mathrm{M}_{2}+1, \mathrm{Q}_{2}}
\end{array}\right] \mathrm{f}\left(\mathrm{y}^{\prime}\right) \mathrm{dy^{ \prime }}=\mathrm{g}\left(\mathrm{x}^{\prime}\right)
\end{aligned}
$$

has its solution given by

$$
\begin{aligned}
& f\left(x^{\prime}\right)=\frac{\lambda x^{\prime \mu^{\prime}-1}}{2 \pi i} \operatorname{Lim}_{\gamma \rightarrow \omega} \int_{c-i \gamma}^{c+i \gamma} x^{\prime-s}\left[\sum_{v^{\prime}=0}^{n} \sum_{u^{\prime}=0}^{v^{\prime}} \frac{\left(-v^{\prime}\right)_{u^{\prime}}\left(-\alpha-r u^{\prime}\right)}{u^{\prime} ! v^{\prime} !} \beta^{v^{\prime}} z^{r v^{\prime}-n}\right] \\
& \sum_{R=0}^{[N / M]} \frac{(-N){ }_{M_{R}}}{R !} A_{1, N, R} \sum_{G=0}^{\omega} \sum_{g^{\prime}=1}^{M_{1}} \frac{(-1)^{G}}{G ! F_{g^{\prime}}} \xi\left(\eta_{G}^{\prime}\right) z^{\prime} \eta_{G}^{\prime} \\
& \times \phi_{3}\left[\frac{-\rho^{\prime} \mathrm{rv}^{\prime}+\rho^{\prime} \mathrm{n}-\mathrm{s}-\sigma^{\prime} \eta_{\mathrm{G}}^{\prime}-\mathrm{R}}{\lambda}\right]\left[\mathrm{z}^{\prime \prime}\left(\frac{-\sigma^{\prime} \eta_{\mathrm{G}}^{\prime}-\rho-\rho \mathrm{rv}+\rho^{\prime} \mathrm{n}-\mathrm{R}}{\lambda}\right)\right]^{-1} \phi(\mathrm{s}) \mathrm{ds}
\end{aligned}
$$

(2) The results obtained by H.M. Srivastava and R.K. Raina [20] follow as special cases of our result on assigning certain values to parameter in the function involve.

(3) Letting $\left.\mathrm{j}=1, \ldots, \mathrm{N}_{2}\right)=\mathrm{B}_{2 \mathrm{j}}\left(\mathrm{j}=\mathrm{M}_{2+1}, \ldots, \mathrm{v}_{2}\right)=1, \sigma^{\prime} \rightarrow 0$ in $(2.1)$ and $\mathrm{n}=\mathrm{q}=\mathrm{g}=\mathrm{B}_{2}=0$ and $\ell$ $=\mathrm{c}=-1$, and $\mathrm{A}_{2}=1$, the result reduces to a known result derived by Chaurasia, V.B.L. and Patni, Rinku [4] with $\mathrm{n}=0$.

(4) Taking $A_{2 j}\left(j=1, \ldots, N_{2}\right)=B_{2 j}\left(j=M_{2}+1, \ldots, \theta_{2}\right)$ and $\sigma^{\prime} \rightarrow 0$ in (2.1), we find a known result of Goyal, S.P. and Mukherjee, Rohit [8] with $\mathrm{t}=0$.

\section{Conclusion}

The Weyl type integral evaluated and the results obtained in this paper are of a general character and may prove to be useful in several interesting situations appearing in the literature on applied mathematics and mathematical physics.

\section{REFERENCES}

[1] Agarwal. R. P., Certain fractional q-integrals and q-derivatives, Proc. Cambridge Philos. Soc., 66(1969), 365-370.

[2] Al-Salam. W. A., Some fractional q-integrals and q-derivatives, Proc. Edin. Math. Soc., 15(1966), 135-140.

[3] Brafman.F., Some generating functions for Laguerre and Hermite polynomials, Canad. J. Math. 9 (1957), 80-187.

[4] Chaurasia. V.B.L and Patni. R., Integral equations of Fredholm type with special functions, Indian J. Pure appl. Math., 32(5), (2001), 747

[5] Chaurasia. V.B.L. and Shekhawat.A.S., Fredholm type integral equations and certain polynomials, Kyungpook Math. J., 45, (2005), 471-481. 
[6] Erdélyi.A., et al., Tables of integral transforms, McGraw-Hill, New York, (1954).

[7] G. Gasper. and M. Rahman., Basic hypergeometric series, Cambridge University Press, Cambridge, 1990.

[8] Goyal. S.P. and Mukherjee. R., on the Laplace Transform and the generalized weyl fractional integral operator involving H-Function, Kyungpook Math.Journal., 44(4),(2004), 481-493.

[9] Inayat and Hussain. A.A., New Properties of hypergeometric series derivable from Feynmann integrals II, A generalization of H-Function, J.Physics.A.: Math.Gen.20(1987), pp.4119-4128.

[10] Jackson. F. H., On q-definite integrals, Quart. J. Pure. Appl. Math., 41(1910), 193-203.

[11] Lahiri. M., On a generalization of Hermite Polynomials, Proc. Amer. Math. Soc., 27, (1971), 117-121

[12] S. G. Samko., A. A. Kilbas. and O. I. Marichev., Fractional Integrals and Derivatives. Theory and Applications, Gordon and Breach Science Publishers, Yverdon, Switzerland, 1993.

[13] Saxena. R. K. and Kumar. R., Recurrence relations for the basic analogue of H-function, J. Nat. Acad. Math., 8(1990), 48-54.

[14] Saxena. R.K., Mathai. A.M., and Haubold. H.J., Reaction-diffusion systems and non linear waves, Astrophysics and space science, Vol 305(2006), 297-303.

[15] Saxena. R.K.and Mathai. A.M., Generalized hypergeometric functions with applications in statistics and physical sciences, Springer-Verlag, Berlin, 1973.

[16] Saxena. R.K.and Mathai. A.M., The H-function with applications in statistics and other disciplines, John Wiley and Sons. Inc. New York, 1978.

[17] Saxena. R. K., Modi. G. C. and Kalla. S. L., A basic analogue of Fox's H-function, Rev. Tec. Ing. Univ. Zulia, 6(1983), 139-143.

[18] Singh. R.P. and Srivastava. K.N., A note on generalization of Laguerre and Humbert polynomials, Ricerca (Napoli) (2) 14 (1963), 11-21, Errata, ibid. 15 (1964), 63.

[19] Spibiñsi. P., Some expansion theorems for the H-function, Ann. Polon. Math., 23,(1970), 125.

[20] Srivastava. H.M. and Raina. R.K., On certain methods of solving a class of integral equations of Fredholm type, The journal of the Australian Mathematical Society,Vol.52 (Series A), (1992), 110.

[21] Srivastava. H.M and Singhal. J.P., A class of polynomials defined by generalized Rodrigues formula, Ann. Mat. Pure Appl., 90 (1971), 75.

[22] Srivastava. H.M. and Singh. N.P., The integration of certain products of Multivariable Hfunction with general class of polynomials, Rend. Circ. Mat.Palermo , (2) 32 (1983), 157-187.

[23] N. Südland., B. Bauman and T.F. Nonnenmacher., Open problem : who know about the Aleph functions? Fract. Calc. Appl. Anal. 1(4) (1998), pp 401-402.

\section{AUTHORS' BIOGRAPHY}

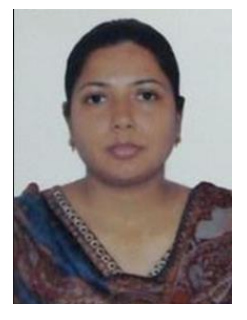

Parul Gupta, Completed PG in Mathematics from University of Kota. Presently pursuing Ph.D in Mathematics from Suresh Gyan Vihar University, Jaipur, Rajasthan under the Guidance of Coauthor Dr Ashok Singh Shekhawat.

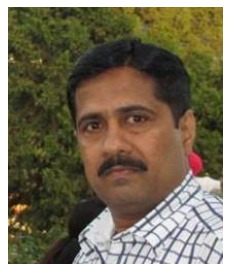

Dr Ashok Singh Shekhawat, He has completed Doctorate under the Supervision of Dr V B L Chourasia, Department of Mathematics, University of Rajasthan, Jaipur. Presently working as a Professor and Head of the Department of Mathematics, Suresh Gyan Vihar University, Jaipur- Rajasthan. 
Citation: P.Gupta, A.S. Shekhawat, "Weyl Fractional Integrals associated with the Transcendental Functions ", International Journal of Scientific and Innovative Mathematical Research, vol. 5, no. 5, p. 9, 2017., http://dx.doi.org/10.20431/2347-3142.0505002

Copyright: (C) 2017 Authors. This is an open-access article distributed under the terms of the Creative Commons Attribution License, which permits unrestricted use, distribution, and reproduction in any medium, provided the original author and source are credited. 